\title{
Preface
}

2018 International Conference on Smart Materials, Intelligent Manufacturing and Automation (SMIMA 2018) was aim at promoting interactions among professionals from the field of Materials and Manufacturing. The SMIMA Committee have invited 192 experts, scientists, researchers and scholars from both academia and the industry to the conference.

SMIMA 2018 was held in School of Information and Control, Nanjing University of Information Science \& Technology, China during May 24-26, 2018. 65 invited speeches and 180 paper presentations were presented by experts and research team leaders from all over the world during the conference. The participants shared their research results and discussed the development and research applications in this field.

The conferences invited submission of researches focus on intelligent manufacturing. Accepted papers after proper registration and presentation will be published into conference proceedings, which will be submitted for index by Ei Compendex and Scopus. Eventually, we received more than 300 articles whose topics include but not limited to Modeling, analysis, and simulation of Intelligent manufacturing, Automation and Nontraditional manufacturing and Digital Signal and Image Processing. These papers were carefully peer reviewed by our international reviews. Each article was reviewed by at least 2 reviewers.

We are very grateful to all the contributors, keynote speakers, presenters and reviews. Your support made this conference a successful event. We would also like to express our gratitude to the co-sponsor of Nanjing University of Information Science \& Technology, China and University of Information Technology, Vietnam.

The Organizing Committee of SMIMA 2018 


\section{Advisory Chair}

Prof.Dr.Jinzhong Min, Nanjing University of Information Science \& Technology(Vice-president)

Prof. Guanghsu Chang, Bakersfield College, USA

\section{Conference Chairs}

Prof.Qingshan Liu, School of Information and Control, Nanjing University of Information Science \& Technology, China(dean)

Prof. Phuoc Vinh Tran, University of Information Technology, Vietnam

Prof.Joseph Heled, Department of Computer Science, University of Auckland, Auckland, New Zealand

\section{Local Chair}

Prof.Anfu Yuan, School of Information and Control,Nanjing University of Information Science \& Technology, China

Dr. Ying Han, School of Information and Safety Engineering, Zhongnan University of Economics and Law, China

\section{Technical Committee}

Prof.A. M. Harsha S. Abeykoon, Asian Institute of Technology, Thailand

Prof.Muralindran Mariappan, Universiti Malaysia Sabah, Malaysia

Dr.Gang Qin, University of Electronic Science and Technology of China, China

Assoc.Prof.Xiaoxia Wang, North University of China, China

Dr.Guanjun Wang, China University of Mining and Technology, China

Dr.Mufeed Batarseh, Abu Dhabi Polytechnic, UAE

Dr.Yousef Haik, Qatar University, Qatar

Prof.Chil-Chyuan Kuo, Ming Chi University of Technology, Taiwan

Prof. İsmail R akıp Karaş, Karabuk University, Turkey

Prof. Vasile Avram, Bucharest University of Economic Studies, Romania

Prof. Chengwen Tan, Beijing Institute of Technology, China

Dr. Zhang Tingyu, Aerospace research institute of materials \& processing technology, China

Prof.Xiaoming You, Shanghai University of Engineering Science, China 